\title{
Uso de kits experimentales para mejorar las actitudes y bajar la repitencia en Química General ${ }^{1}$
}

\author{
Use of experimental kits to improve attitudes \\ and lower repetition in general chemistry
}

\section{Uso de kits experimentais para melhorar as atitudes e menor repetição na química geral}

\author{
M. F. Molina \\ Recibido: febrero 26 de 2018 - Aceptado: junio15 de 2018
}

\begin{abstract}
Resumen- El presente artículo expone una propuesta de innovación de aula, incorporando la actividad experimental en clases magistrales por medio del uso de kits didácticos para mejorar las actitudes negativas de los estudiantes universitarios hacia la química y bajar la repitencia en un curso de química general. Se aplicaron 8 kits, con su desarrollo, elaboración de guías y utilización, obteniendo resultados positivos sobre dichas actitudes hacia la química, considerando su dificultad, el interés del conocimiento químico y la obtención de un aprendizaje disciplinar adecuado. Adicionalmente, los kits permitieron bajar el índice de pérdida del curso al 5\%, comparado con el $38 \%$ que se presentaba antes, en los cursos de Química General en la Universidad Nacional de Colombia.
\end{abstract}

Palabras clave - actitudes, innovación, kits, química general, repitencia.

Abstract - This article develops a classroom innovation proposal, incorporating experimental activity in master classes through the use of didactic kits to improve negative attitudes

Proyecto de investigación: código: 36588. Código quipu: 902010125021. Uso de kits para convertir el aula tradicional en un laboratorio de química. Convocatoria investigación sobre innovación pedagógica sede Bogotá. Universidad Nacional de Colombia 2016.

M. F. Molina, Universidad Nacional de Colombia, Bogotá, Colombia, email:mfmolinac@unal.edu.co

Como citar este artículo: Molina, M. F. Uso de kits experimentales para mejorar las actitudes y bajar la repitencia en química general, Entre Ciencia e Ingeniería, vol 12, no. 24, pp. 89-95, julio-diciembre 2018. http://dx.doi.org/10.31908/19098367.3817

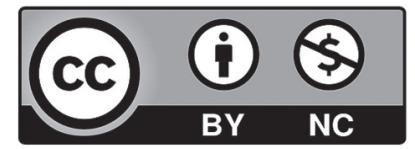

Attribution-NonCommercial 4.0 International (CC BY-NC 4.0) toward chemistry and lower repetition in a general chemistry course. It is possible to apply 8 kits, with their development, development of guides and use, giving positive results on the attitudes towards chemistry, considering its difficulty, the interest of chemical knowledge and obtaining an appropriate disciplinary learning. Additionally, the kits allowed obtaining a rate of loss of the course over $5 \%$ compared to $38 \%$ for the General Chemistry courses at the National University of Colombia.

Key Words - attitudes, innovation, kits, general chemistry, repetition.

Resumo - Este artigo desenvolve uma proposta de inovação em sala de aula, incorporando atividade experimental em master classes através do uso de kits didáticos para melhorar as atitudes negativas em relação à química e menor repetência em um curso de química geral. É possível aplicar 8 kits, com o seu desenvolvimento, desenvolvimento de guias e uso, dando resultados positivos nas atitudes frente à química, considerando sua dificuldade, o interesse do conhecimento químico e a obtenção de um aprendizado disciplinar adequado. Além disso, os kits permitiram obter uma taxa de perda do curso superior a $5 \%$ em comparação com $38 \%$ dos cursos de Química Geral da Universidade Nacional da Colômbia.

Palavras chave - atitudes, inovação, kits, química geral, repetição.

\section{INTRODUCCIÓN}

$\mathrm{L}$ AS actitudes hacia la química, particularmente al considerarla difícil de aprender, se muestran algunas veces negativas y limitan la enseñanza de esta ciencia [1], situación preocupante porque, de entrada, sin haber accedido a su enseñanza, se asume complejo su aprendizaje. Por esta razón, se convierte en un reto el hecho de cambiar las 
actitudes negativas de los estudiantes ante la dificultad, sin bajar la exigencia y sin disminuir la calidad de enseñanza, dentro de un curso de Química General a nivel universitario, para que como resultado social se presente un bajo índice de pérdida del curso.

Confrontar el problema de las actitudes hacia la ciencia con métodos novedosos de enseñanza es un trabajo que han venido desarrollando las investigaciones en didáctica [2]. Las dificultades didácticas y metodológicas en la enseñanza de la química se reconocen y por ello, en los estudios se leen críticas sobre la enseñanza tradicional de esta, y se proponen diversas metodologías, como el aprendizaje cooperativo, la enseñanza activa, etc., pero en la realidad la situación dentro del aula poco cambia, muchas de estas ideas se quedan para los congresos, y al volver al aula el docente sigue actuando tradicionalmente y sin innovaciones.

En este sentido, es posible que exista una desconexión entre las metodologías y los recursos que se emplean en el proceso de enseñanza, razón por la cual las propuestas se quedan en el aire. Partiendo de esta situación, el presente trabajo ofrece la posibilidad de realizar cambios metodológicos con materiales que pueda utilizar el profesor en el aula de clase; estos son los kits de actividades experimentales de aula que, al ser utilizados dentro de un salón de clase, lo convierten momentáneamente en un laboratorio experimental, sirviendo además como un motivante del aprendizaje.

\section{EL USO DE LOS KITS}

Un kit de ciencia se define como una agrupación de materiales e instrucciones, empaquetados y usados como una herramienta de instrucción para mejorar la comprensión de la ciencia [3]. Estos ofrecen la posibilidad de generar espacios de enseñanza formales e informales, de tal forma que permiten enseñar la ciencia tanto a expertos como a novatos.

Los kits han mostrado ser efectivos en la motivación, con un impacto positivo en las actitudes científicas [3], tanto de estudiantes como de profesores, útiles para enseñar contenidos, con un efecto positivo en la adquisición de conocimiento [4]. Han sido utilizados de forma comercial y no comercial por décadas, ya que ayudan a generar un aprendizaje activo y, a la vez, son herramientas para apoyar la enseñanza por indagación [5].

Dichos kits permiten ir más allá de la enseñanza de un contenido, involucrar a los estudiantes en trabajo activo de aula y en la resolución cooperativa de problemas experimentales a pequeña escala [6]. Pueden mejorar el aprendizaje, porque contribuyen a mostrar que los conceptos abstractos de química en el tablero poseen aplicaciones con relevancia para la sociedad. Así, los kits son una alternativa de material de clase que pueden ser utilizados y estudiados para fundamentar su uso didáctico.

Un paso crítico en el empleo de los kits es su implementación, ya que los que se venden comercialmente poseen un contexto mayoritariamente de país desarrollado, o tienen en las guías de trabajo una metodología de indagación que no es posible aplicar en todos los lugares. Incluyen materiales para más de una persona o para un grupo determinado, no permiten a veces la sustitución de consumibles o han sido probados por expertos que colocan una exigencia superior a la de un contexto particular [3]. Por ello, trabajar en la implementación de kits para un contexto dado se convierte en una labor de importancia didáctica, con grandes posibilidades de mejorar los entornos de aula, las actitudes hacia la ciencia y la pedagogía de conocimiento del contenido [5].

Por esta razón, el desarrollo de los kits requiere investigación, además de financiación, para formar a los docentes en metodologías de enseñanza activa, en la construcción de materiales desde la consecución de la materia prima hasta la estructuración de un kit con todas sus partes, que logre que sea funcional y adaptable a sitios donde no es posible reponer consumibles fácilmente. Es importante pasar de la disertación sobre los problemas en la enseñanza de las ciencias a la implementación en el terreno de propuestas con metodologías motivantes que traten de resolver los problemas actuales en la enseñanza de las ciencias [7].

Propósito del trabajo: El propósito de este estudio es determinar el efecto del uso de kits experimentales en el aprendizaje y las actitudes hacia la química en un curso de Química General.

Problema: Las actitudes negativas hacia la dificultad de la química se convierten en un obstáculo de superación de un curso de química general y en causal de deserción universitaria.

\section{MetodologíA}

Se utilizó la investigación-acción como marco de este trabajo, considerando que se parte de una problemática de aula que se buscó modificar por medio de una propuesta de innovación didáctica. Se sigue el planteamiento de las fases, de planificación (el conocimiento de la problemática, las temáticas y las actitudes de los estudiantes), actuación (con el desarrollo de los materiales y la posterior aplicación), observación (encuestas, entrevistas, actitudes hacia el desarrollo de la aplicación) y finalmente, la reflexión (de los resultados obtenidos, como insumo de un nuevo ciclo de investigación) [8].

El desarrollo de este proyecto comprendió las siguientes fases:

1. Conocimiento de las actitudes hacia la química del grupo de aplicación, por medio de un test ya estandarizado [1].

2. Diseño y desarrollo de 8 kits didácticos ( 6 originales y 2 híbridos con los obtenidos comercialmente) para trabajar los temas de estequiometría, reacciones en medio acuoso, cinética química, equilibrio químico, equilibrio ácidobase, agua y sus propiedades y estructura molecular.

3. Validación del uso de los kits. La utilización de los kits siguió una guía que consideró la enseñanza por indagación como componente didáctico. La validación se hizo por medio de un test de actitudes hacia el trabajo con kits.

4. Realización de una prueba de preconceptos para medir el aprendizaje logrado con el uso de cada kit. 
5. Aplicación de los kits con el grupo de estudio.

6. Evaluación de la propuesta, desde las actitudes desarrolladas y el porcentaje de pérdida del curso.

\section{A. Población}

El proyecto de innovación de aplicó a 56 estudiantes del curso de Principios de Química en el segundo semestre de 2017, en la Universidad Nacional de Colombia-Bogotá; todos los estudiantes pertenecen a la carrera de Ingeniería Agrícola, correspondiente a 16 mujeres y 40 hombres con edades entre los 16 y los 19 años.

\section{B. Propuesta}

Aplicaciones: Durante el periodo de 16 semanas que dura el curso se utilizaron 8 kits, distribuidos en las diferentes sesiones. Cada kit fue acompañado de una guía de aprendizaje por indagación para resolver durante la sesión de 100 minutos. El kit se utilizó en una sesión posterior a la clase teórica sobre la temática, y funcionó como material para adelantar una actividad practica de los conceptos estudiados. Luego del uso del kit se aplicó un pos-test de preconceptos donde aparecen concepciones sobre el tema y el test de actitudes hacia el uso del kit (Tabla I).

Listado de Kits:

1. Medidas

2. Vitamina $\mathrm{C}$

3. Reacciones

4. Dióxido de carbono

5. Agua

6. Equilibrio

7. $\mathrm{pH}^{*}$

8. suelos*

*estos kits no tuvieron aplicación del test de actitudes hacia el Kit debido a que son híbridos entre comercial y elaborado.

\section{Resultados}

En la Fig. 1 se aprecian los kits, los cuales se elaboraron en cubetas de $500 \mathrm{~mL}$ y $1000 \mathrm{~mL}$ (Kit de dióxido de carbono). Están compuestos de reactivos muy diluidos en goteros; los materiales típicos de laboratorio fueron reemplazados así: una jeringa hace las veces de una pipeta o bureta; los goteros son dispensadores que pueden parecer una bureta si se mide el volumen de una gota; los erlenmeyer o vasos son reemplazados por copas de 1 onza; los agitadores son reemplazados por palillos de café; los tubos de ensayo fueron tubos de escarcha; los balones con desprendimiento fueron tubos con desprendimiento, y en caso de requerir saber masas, estas fueron medidas con anterioridad. Los desechos generados fueron mínimos y se colectaron en un recipiente para su adecuada disposición.

A continuación se observan algunas imágenes del trabajo con los kits (fig. 2, 3 y 4). Se nota el espacio en el cual se trabajó, no adaptado para actividades en grupo y que limita la interacción entre estudiantes.

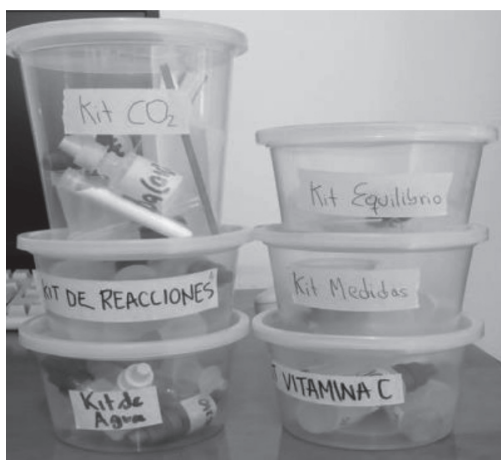

Fig. 1. Vista de los kits desarrollados.

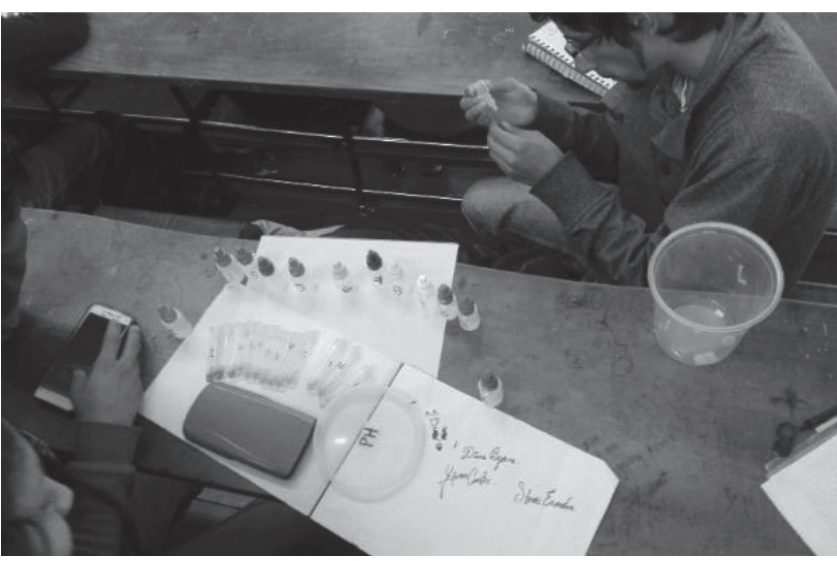

Fig. 2. Trabajo con kit de pH.

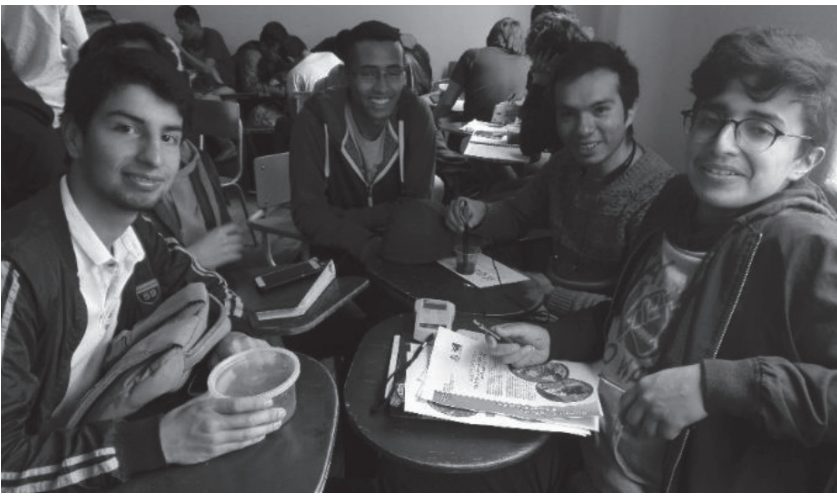

Fig. 3. Trabajo con kit de suelos.

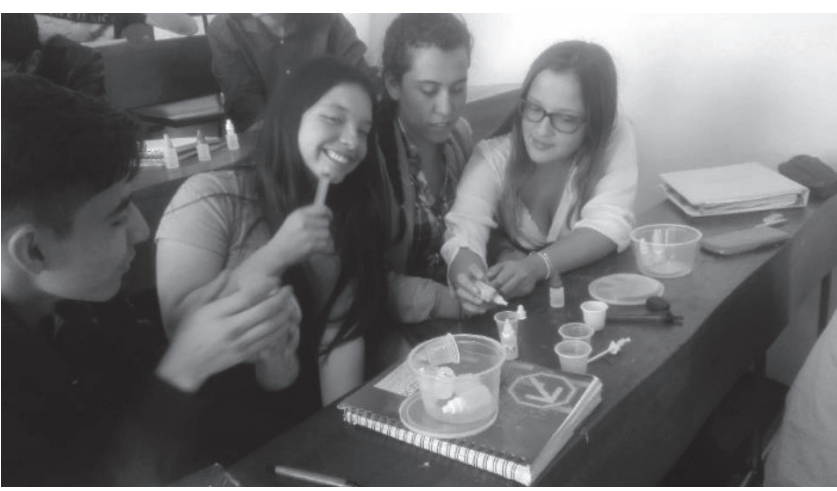

Fig. 4. Trabajo con kit de vitamina $C$.

Para cada kit se aplicó el test 1 de actitudes hacia los kits. Este test fue adaptado de Foley [9], y se tabuló como aparece a continuación en la Tabla I. El test indaga sobre la aprobación que puede tener el uso de cada kit limitado 
a la actividad práctica, y no aborda el aprendizaje que se adquirió con el uso del mismo, ya que este aprendizaje se determina con el test de preconcepciones, resultados que están publicados para el kit de vitamina $\mathrm{C}$ en la ponencia presentada por Molina [10] en el 52 Congreso Mexicano de Química-2017.

\section{A. Test 1 de actitudes sobre el uso del kit.}

Lee atentamente cada enunciado y señala tu respuesta con una $\mathbf{X}$, en el cuadro respectivo. Posees las siguientes opciones: $\mathbf{T A}=$ totalmente de acuerdo (5). $\mathbf{A}=$ de acuerdo (4). $\mathbf{I}=$ No estoy seguro (a). Indecisión, ni fu ni fa (3) $\mathbf{D}=$ en desacuerdo (2). $\mathbf{T D}=$ totalmente en desacuerdo (1).

TABLA I

RESULTADOS DE ACTITUDES HACIA LOS KITS

\begin{tabular}{|c|c|c|c|c|c|c|c|c|}
\hline ítem & $\begin{array}{c}\text { Pregunta/ Kit } \\
\text { y promedio }\end{array}$ & $\mathbf{1}$ & $\mathbf{2}$ & $\mathbf{3}$ & $\mathbf{4}$ & $\mathbf{5}$ & $\mathbf{6}$ & Promedio \\
\hline 1. & $\begin{array}{c}\text { Utilizar el kit } \\
\text { fue un proceso } \\
\text { sencillo. }\end{array}$ & 4,8 & 4,3 & 4,3 & 4,3 & 4,4 & 4,3 & 4,4 \\
\hline 2. & $\begin{array}{c}\text { La guía del kit } \\
\text { fue sencilla de } \\
\text { seguir. }\end{array}$ & 4,6 & 4,1 & 4,0 & 4,2 & 4,2 & 4,3 & 4,2 \\
\hline 3. & $\begin{array}{c}\text { La asesoría del } \\
\text { profesor fue } \\
\text { útil. }\end{array}$ & 4,5 & 4,6 & 4,4 & 4,5 & 4,5 & 4,4 & 4,5 \\
\hline 4. & $\begin{array}{c}\text { El grupo } \\
\text { disfrutó el uso } \\
\text { del kit. }\end{array}$ & 4,7 & 4,4 & 4,3 & 4,2 & 4,4 & 4,2 & 4,4 \\
\hline 5. & $\begin{array}{c}\text { Recomendaría } \\
\text { utilizar este kit } \\
\text { en otro curso. }\end{array}$ & 4,7 & 4,4 & 4,2 & 4,4 & 4,4 & 4,2 & 4,4 \\
\hline 6. & $\begin{array}{c}\text { Trabajaría otro } \\
\text { kit en el curso } \\
\text { con agrado. }\end{array}$ & 4,8 & 4,6 & 4,4 & 4,6 & 4,6 & 4,4 & 4,6 \\
\hline
\end{tabular}

El promedio general de los 6 ítems para los 6 kits fue de 4,4, lo cual indica actitudes positivas hacia los kits. Importante resaltar que se abre la posibilidad de "trabajar con otro kit en otro momento", en otro curso o lugar, un factor positivo para utilizar kits en otras materias. En la misma tendencia está el ítem 2, con un promedio de 4,2, cuyo valor se presenta un poco por debajo del 4,4 general, resultado que indica quizás que la guía debe ser revisada y encuentra reparos por parte de la población.

Las guías conducen al trabajo activo por indagación, lo cual no es comúnmente utilizado por los estudiantes; la indagación les obliga a ver que no hay respuestas fáciles y, por tanto, puede aparecer el desánimo; sin embargo, esta metodología logra mayores rendimientos frente a la tradicional [11]. La novedad de la metodología de indagación abre un espacio de relevancia para el trabajo del profesor, dándole una nota alta, 4,5, con la que se manifiesta un acuerdo con el trabajo realizado, que consistió en apoyar con sugerencias, preguntas e indicaciones para direccionar la actividad. Todo el grupo logra completar la guía y presentar el resultado sugerido. Para terminar este análisis, es importante resaltar la sencillez de la utilización de los kits, en su mayoría adición de reactivos, y el disfrute del trabajo realizado con estos.

\section{B. Actitudes hacia la Química}

Las actitudes hacia la enseñanza de las ciencias, de la química en particular para este estudio, están relacionadas con el nivel de aprendizaje [12], y determinan la apropiación de estos conocimientos para participar en sociedad. Son importantes en la comprensión de los conceptos científicos, formando incluso la capacidad de explicar los fenómenos cotidianos [13]. Las actitudes consideran la suma total de inclinaciones, sentimientos, prejuicios, nociones preconcebidas, temores, amenazas o convicciones del individuo acerca de un asunto determinado, por lo cual son un parámetro psicológico que influye en el aprendizaje, ya que genera una respuesta positiva o negativa en el desarrollo social en general [14].

TABLA II

TeSt 2 DE ACTITUdES HACIA LA QUímica

Carrera--------------Semestre---Edad--Sexo: F--- / M---

\begin{tabular}{|c|c|c|c|c|c|c|}
\hline 1. & $\begin{array}{l}\text { El curso de química me parece } \\
\text { más agradable que otros cursos. }\end{array}$ & TA & A & I & $\mathrm{D}$ & TD \\
\hline 2. & $\begin{array}{l}\text { Los símbolos utilizados en la } \\
\text { clase de química me parecen } \\
\text { difíciles de entender. }\end{array}$ & TA & A & I & $\mathrm{D}$ & $\mathrm{TD}$ \\
\hline 3. & $\begin{array}{l}\text { Me gustaría tener clases de } \\
\text { química con mayor frecuencia. }\end{array}$ & TA & A & I & $\mathrm{D}$ & $\mathrm{TD}$ \\
\hline 4. & $\begin{array}{l}\text { La química sirve para conocer } \\
\text { muchos aspectos de nuestra vida } \\
\text { cotidiana. }\end{array}$ & TA & A & I & $\mathrm{D}$ & $\mathrm{TD}$ \\
\hline 5. & $\begin{array}{l}\text { Resuelvo con facilidad los } \\
\text { problemas de química. }\end{array}$ & TA & A & I & $\mathrm{D}$ & TD \\
\hline 6. & $\begin{array}{l}\text { Me aburro durante las clases de } \\
\text { química. }\end{array}$ & TA & A & I & $\mathrm{D}$ & $\mathrm{TD}$ \\
\hline 7. & $\begin{array}{l}\text { El desarrollo de la química } \\
\text { mejora nuestra calidad de vida. }\end{array}$ & TA & A & I & $\mathrm{D}$ & $\mathrm{TD}$ \\
\hline 8. & $\begin{array}{l}\text { La esperanza de resolver muchos } \\
\text { problemas ambientales está en la } \\
\text { química. }\end{array}$ & TA & A & I & D & $\mathrm{TD}$ \\
\hline 9. & $\begin{array}{l}\text { La química es una ciencia } \\
\text { muy compleja para mi nivel } \\
\text { académico. }\end{array}$ & TA & A & I & $\mathrm{D}$ & $\mathrm{TD}$ \\
\hline 10. & $\begin{array}{l}\text { El lenguaje de la química y sus } \\
\text { símbolos son fáciles de entender. }\end{array}$ & TA & A & I & $\mathrm{D}$ & $\mathrm{TD}$ \\
\hline 11. & $\begin{array}{l}\text { La profesión de químico es poco } \\
\text { interesante. }\end{array}$ & TA & A & I & $\mathrm{D}$ & $\mathrm{TD}$ \\
\hline 12. & $\begin{array}{l}\text { Todas las carreras de la } \\
\text { universidad deberían tener un } \\
\text { curso de química. }\end{array}$ & TA & A & I & $\mathrm{D}$ & $\mathrm{TD}$ \\
\hline 13. & $\begin{array}{l}\text { Los conocimientos en química } \\
\text { son necesarios en el desarrollo } \\
\text { de mi carrera. }\end{array}$ & TA & A & I & $\mathrm{D}$ & $\mathrm{TD}$ \\
\hline 14. & $\begin{array}{l}\text { Desearía tener pocas clases de } \\
\text { química. }\end{array}$ & TA & A & I & $\mathrm{D}$ & TD \\
\hline 15. & $\begin{array}{l}\text { Comprendo los conceptos de } \\
\text { química con facilidad. }\end{array}$ & TA & $\mathrm{A}$ & I & $\mathrm{D}$ & TD \\
\hline 16. & $\begin{array}{l}\text { El curso de química es muy } \\
\text { interesante. }\end{array}$ & TA & A & I & $\mathrm{D}$ & TD \\
\hline
\end{tabular}


En la tabla III aparecen los resultados del test de actitudes (Tabla II) hacia la química, aplicado al inicio y al final de curso (semanas 2 y 14 de un curso de 16 semanas). El test corresponde a 16 ítems, 5 escritos de forma negativa y $11 \mathrm{de}$ forma positiva valorados de 1 a 5 . En el caso de los ítems negativos se invierte la escala para presentarlos todos de forma positiva. Los ítems presentados de forma negativa corresponden al 2, 6, 9, 11 y 14 .

Los ítems 4 y 7 son los de mayor valor, 4,7 y 4,6 respectivamente, correspondiente a actitudes muy positivas. Las dos afirmaciones están relacionadas con la química fuera del aula, la que se ve en los medios y en la vida cotidiana, y tienen que ver con el impacto directo de la química, como son los medicamentos, los materiales, los productos de limpieza y aseo, los alimentos, etc., aspecto valorado por la población en general, y que en otros estudios ha mostrado resultados similares [15]. A estos ítems también se puede unir el $8(4,4)$ para integrarlos en una categoría que es posible designar como "actitudes hacia la química fuera del aula"; son aquellas actitudes que desarrolla el ciudadano sobre la química, aun cuando no ha tenido contacto directo con esta ciencia. La química fuera del aula tiene como promedio general 4,6, inicialmente de 4,4; el leve cambio indica que estas concepciones no dependen mucho del trabajo de aula y permanecerán seguramente así, independientemente de la metodología de clase aplicada.

TABLA III

ACTITUdes HACIA LA QUímiCA
\begin{tabular}{|c|c|c|}
\hline Ítem & Semana 2 & Semana 14 \\
\hline 1 & 3,5 & 4,1 \\
\hline 2 & 3,3 & 3,5 \\
\hline 3 & 3,2 & 3,7 \\
\hline 4 & 4,5 & 4,7 \\
\hline 5 & 2,9 & 3,6 \\
\hline 6 & 3,6 & 4,0 \\
\hline 7 & 4,5 & 4,6 \\
\hline 8 & 4,2 & 4,4 \\
\hline 9 & 3,4 & 3,7 \\
\hline 10 & 3,2 & 3,9 \\
\hline 11 & 3,7 & 3,8 \\
\hline 12 & 3,2 & 3,8 \\
\hline 13 & 4,1 & 4,3 \\
\hline 14 & 3,5 & 3,7 \\
\hline 15 & 3,0 & 3,5 \\
\hline 16 & 3,8 & 4,3 \\
\hline
\end{tabular}

Una comparación que debe hacerse es entre las respuestas del inicio del curso y las del final. Las respuestas iniciales no valoran el desarrollo del curso, tienen que ver tal vez con las experiencias obtenidas en cursos anteriores de química, principalmente los de colegio. Los tres ítems de la categoría presentan cambios similares: un aumento entre 0,1 y 0,2 unidades. Esta categoría a nivel de colegio, colocada como "ciencia fuera de la escuela", tiene un promedio final entre $5^{\circ}$ y $11^{\circ}$, de 3,8 para mujeres, y 3,8 para hombres [2]. Se observa que la actitud es más positiva en esta población universitaria, posiblemente porque es seleccionada durante el examen de admisión como parte de una "elite académica" que conoce más acerca de la ciencia. Se puede considerar que el efecto de la metodología en esta categoría es mínimo, probablemente porque la Química fuera del aula depende más de los medios de comunicación, cuestión a la que están más expuestas las nuevas generaciones. Por ello, esta categoría no es la adecuada para discutir el efecto del proyecto de innovación con kits.

Otro contraste necesario es entre los ítems altos y los bajos. Dentro de estos últimos se encuentra principalmente el ítem 15 con un valor de 3,5 que está asociado con los ítems $2,5,9,10$, los cuales se clasifican en la categoría "dificultad" hacia la química. Molina et al. [1] encontraron hacia esta categoría en estudiantes universitarios de diferentes carreras, un promedio de 3,2, frente a valores que oscilan entre 3,5 y 3,9. Los ítems 5 y 15 presentan en el pretest los valores más bajos, 2,9 y 3,0, relacionados con la facilidad para resolver y aprender conceptos de química. Las actitudes se orientan hacia negativas y aumentan luego de la aplicación del proyecto, hacia positivas de forma leve, 3,6 y 3,5. Aunque el aumento es mínimo, puede ser significativo si se compara con el 3,2 encontrado en la cita anterior; de esta forma, las actitudes hacia dificultad de la química se convierten en un factor importante de consideración al proponer proyectos de mejoras didácticas que busquen la superación del fracaso en química [16].

El promedio de la categoría "dificultad" es inicialmente de 3,2 y pasa a 3,6 , con un cambio que parece insignificante. Ante esto, puede pensarse que el proyecto no aportó mayor relevancia a la dificultad que se percibe hacia la química. Una hipótesis al respecto es que la actitud hacia la dificultad es intrínseca, producto del ambiente, relacionado con un autoconcepto de ciencia, el cual Molina et al. [2] encontraron con un valor promedio de 3,7 en estudiantes de secundaria de $5^{\circ}$ a $11^{\circ}$. Tal autoconcepto se convierte en una idea alternativa difícil de modificar en un solo curso, ya que se ha adquirido un bagaje alrededor de la ciencia que la excluye del futuro de sus actividades (3,2 de promedio para el mismo estudio). Dicha situación es quizás una alerta para trabajar en el concepto de ciencia durante la formación de los escolares, independientemente de si elegirán carreras científicas en el futuro, para que se cambie la percepción de que su estudio es difícil y al que solo accede cierto tipo de personas con características especiales.

La agrupación de los ítems 1, 3, 6, 14 y 16 dentro de la categoría interés, resalta que los ítems 1 y 16 presentaron una calificación positiva, de 4,1 y 4,3 respectivamente, con aumentos de 0,6 y 0,5. Ambas afirmaciones están relacionadas directamente con la valoración sobre el desarrollo del curso, resaltando que es agradable e interesante. Aunque el estudiante percibe que la química es difícil, el ambiente 
de clase ayuda a que esté conectado y se involucre en el aprendizaje. Es decir, un estudiante puede llegar a tener resultados similares académicamente sin la metodología de los kits, pero el efecto en su actitud, que puede determinar el aprendizaje a largo plazo, el querer acercarse más a la química por cuenta propia, es más importante que los aprendizajes en sí [17].

Dentro de la anterior categoría, los ítems 3 y 14 que ofrecen la misma información, poseen los valores más bajos, 3,7, lo cual se explica por la relación con la intensidad horaria de las clases, 5 horas semanales, similar a otros cursos, y aunque puede esperarse un no rotundo a más horas de clase, 3,7 manifiesta que por lo menos más de la mitad de los estudiantes no tendría problema con asistir a más horas, y que el resto atiende bien en las que toma. Finalmente, el ítem 6 muestra que no hay una contradicción importante con los ítems 1 y 16, ya que este fue escrito en forma negativa y pueden existir respuestas por interpretación equivocada de la afirmación, lo que hace que tenga 4,0 y no 4,1 o 4,3 como los ítems 1 y 16.

Para cerrar el análisis de actitudes, la categoría que resta es la de "utilidad de la química", dada por los ítems 11, 12 y 13 , con un promedio inicial de 3,7 y uno final de 4,0, que deja con actitudes positivas la utilidad de la química. Llama la atención el cambio de 0,6 unidades en el ítem 12, de 3,2 a 3,8, cercana al cambio más alto de 0,7 en los ítems 5 y 10 , relacionado con la presencia de un curso de química en todas las carreras de la universidad. Si se traduce el valor final en porcentaje, se encuentra que el 76\% (antes 64\%) de los encuestados reconoce la importancia de un curso de química en las diferentes profesiones, y que el efecto del curso con la metodología empleada fue aumentar el valor en un $12 \%$, cuestión que se considera positiva. La valoración de los conocimientos de química dentro de una profesión es un valor positivo para este proyecto, un factor importante del efecto del curso de química a largo plazo.

\section{Repitencia del curso}

Un objetivo importante de este proyecto fue incidir en el porcentaje de repitencia que tiene el curso de Química General, el cual ronda el 38\%, según el documento Pérdida de asignaturas, sede Bogotá, 2013-I [18]. Para el curso donde se aplicó el proyecto, el porcentaje de pérdida estuvo alrededor del $5 \%$, descartando a los estudiantes que desertaron de los 56, esto es, perdieron 3 estudiantes. La metodología contempla el trabajo en equipo durante todo el semestre, cuestión que ayuda a impulsar a los estudiantes rezagados y a motivar el aprendizaje de los conceptos de química que parecen difíciles. Un aspecto relevante de la metodología, es que esta logra una mayor dedicación al estudio de la química y una mejor valoración del aprendizaje, lo cual conduce a este tipo de resultados. Finalmente, los cambios en las actitudes negativas, hacia actitudes positivas, aunque sean leves, inciden inevitablemente en el rendimiento escolar [16].

\section{Conclusiones}

El uso de kits para realizar actividades prácticas dentro de un aula de clase tradicional, permite innovar el proceso de enseñanza de la química, y logra que los contenidos sean más significativos, que se establezcan relaciones entre Ciencia-Tecnología-Sociedad, que se mejoren las actitudes hacia la dificultad y aprendizaje de la química, sobre todo hacia el interés del conocimiento químico, y que se alcance un aprendizaje disciplinar adecuado.

Además, el trabajo realizado incentiva la producción de materiales de enseñanza y la labor docente, ofrece herramientas prácticas para que este construya sus propios kits, con un valor agregado sobre los kits comerciales. Se constituye en un proceso innovador que genera cambios didácticos pertinentes para contrarrestar las actitudes negativas encontradas en estudios sobre la ciencia, y la química en particular. Adicionalmente, los kits permitieron obtener un índice de pérdida del curso sobre el $5 \%$, cuando el valor ronda el 38\% para los cursos de Química General en la Universidad Nacional de Colombia.

\section{REFERENCIAS}

[1] Molina, M. F., Carriazo, J. G. y Farías, D. M. "Actitudes hacia la química de estudiantes de diferentes carreras universitarias en Colombia", Quimica Nova, vol. 34, no. 9, pp. 1672-1677, 2011.

[2] Molina, M. F., Carriazo, J. G. y Casas, J. A., "Estudio transversal de las actitudes hacia la ciencia en estudiantes de grados quinto a undécimo", Tecné, Episteme y Didaxis, no. 33, pp. 103-122, 2013.

[3] Dickerson, D. L., Stewart, C. O., Hathcock, S. y McConnell, W., "The nature and role of science kits in affecting change in public attitude toward understanding of science", In Communicating Science to the Public, pp. 47-62, Springer, Dordrecht, 2014.

[4] Molina, M. F., Palomeque, L. A. y Carriazo, J. G., "Experiencias en la enseñanza de la química con el uso de kits de laboratorio", Entre Ciencia e Ingeniería, vol. 10, no. 20, pp. 76-81, 2016.

[5] Houston, L. S., Fraser, B. J. y Ledbetter, C. E., "An evaluation of elementary school science kits in terms of classroom environment and student attitudes", Journal of Elementary Science Education, vol. 20 no. 4, pp. 29-47, 2008.

[6] López, R. E. y Schultz, T., "Two revolutions in K-8 science education", Physics Today, vol. 54, no. 9, pp. 44-49, 2001.

[7] Dickerson, D. L., Clark, M., Dawkins, K. y Horne, C., "Using science kits to construct content understandings in elementary schools", Journal of Elementary Science Education, vol. 18, no. 1, pp. 43-56, 2006.

[8] Vázquez B., B., Jiménez-Pérez, R. y Mellano J., V., “¿Cómo podemos llevar a cabo una investigación-acción para mejorar la práctica en el aula de ciencias?", Revista Investigações em Ensino de Ciências, vol. 13, no. 1, pp. 45-64, 2008.

[9] Foley, J. M., Bruno, B. C., Tolman, R. T., Kagami, R. S., Hsia, M. H., Mayer, B. and Inazu, J. K., "2C-MORE Science Kits as a Classroom Learning Tool", Journal of Geoscience Education, no. 61, pp. 256267, 2013.

[10] Molina, M. F., "Uso de un kit experimental para enseñar conceptos y actitudes en química general", 52 Congreso Mexicano de Química, Puerto Vallarta, Jalisco, México, 26 al 29 de septiembre de 2017.

[11] Abdi, A., "The Effect of Inquiry-Based Learning Method on Students' Academic Achievement in Science Course", Universal Journal of Educational Research, vol. 2, no. 1, pp. 37-41, 2014.

[12] Raved, L., O. B. Z, "Assaraf. Attitudes towards science learning among 10th-grade students: A qualitative look", International Journal of Science Education, vol. 33, no. 9, pp. 1219-1243, 2011.

[13] Nieswandt, M., "Student Affect and Conceptual Understanding in Learning Chemistry", Journal of Research in Science Teaching, vol. 44, no. 7, pp. 908-937, 2007.

[14] Garritz, A., "Pedagogical Content Knowledge and the affective domain of Scholarship of Teaching and Learning", International Journal for the Scholarship of Teaching and Learning, vol. 4, no. 2, pp. 1-6, 2010 
[15] Barmby, P., Kind, P. M. and Jones, K., "Examining changing attitudes in secondary school science", International journal of science education, vol. 30, no. 8, pp. 1075-1093, 2008.

[16] Salome, C., "The Impact of Students' Attitudes on the Teaching and Learning of Chemistry in Secondary Schools in Bureti District, Kenya", Journal of Emerging Trends in Educational Research and Policy Studies, vol. 4, no. 4, pp. 618-626, 2013.

[17] Simon, S., "Attitudes to Science and to Learning Science", In: Gunstone, R. (eds), Encyclopedia of Science Education, Springer, Dordrecht, 2014.

[18] Rodríguez-Niño, G., Pérdida de Asignaturas, Universidad Nacional de Colombia, Bogotá, [En línea]. Available: https://docs.google.com/

Manuel F. Molina C. Nació en El Colegio (Cundinamarca-Colombia), profesor Asociado del Departamento de Química de la Universidad Nacional de Colombia en Bogotá, donde se graduó de Químico en el 2000, y Magister en Ciencias en el 2003. Estudió una Especialización en Pedagogía en la Universidad Pedagógica Nacional en el 2001. Actualmente enseña Química General en carreras de Ciencias e Ingeniería, y su respectivo laboratorio. Investiga en Enseñanza de la Química y actúa como divulgador de la Ciencia. 